\title{
Multi agent based Framework for Traffic monitoring in VANET
}

\author{
Divya Chadha \\ Maharishi Markandeshwar University \\ Mullana,Ambala \\ Haryana,India
}

\author{
Reena Dahiya \\ Maharishi Markandeshwar University \\ Mullana,Ambala \\ Haryana,India
}

\begin{abstract}
For last many years studies have been carried out to inculcate technical advancement in the field of VANET traffic monitoring systems. Rapidly growing population and number of vehicles have increased the probability of network congestion, traffic jams and road accidents. To overcome these hazardous situations, series of technologies have been implemented on vehicle infrastructure in recent years. Vehicles are equipped with intelligent on board equipments and communication is facilitated between vehicle and roadside units to enhance road safety. In this paper multi agent base framework is proposed to increase coordination and synchronization among vehicles in VANETs.
\end{abstract}

Keywords: Mobile Agent, VANET, Vehicle communicator

\section{INTRODUCTION}

For adding intelligence to vehicles various mechanisms have been proposed so far. Communication between vehicles becomes effective if information sharing and collaboration between nodes is faster. VANET is a subset of MANET. It is necessary for vehicles to communicate with each other and roadside units and share security information and warning alerts. Mobile agents are used in VANETs for attaining this goal. Mobile agents are independent software program that have ability to migrate from one node to another in network during their execution. While migrating and travelling from one node to other mobile agents have capability to collect and deliver information and carry result to multiple locations in network after computations.

Network performance and productivity improves when nodes are equipped with high computational power. Mobile agents can efficiently perform computational task $\mathrm{s}$ in dynamic network environment and distributed applications.

Vehicles participating in VANETs have to communicate with one another for sharing various types of information and alerts. For effective communication it is necessary for all the vehicle nodes to participate in communication, respond to neighboring vehicles and follow instructions according to alerts and warnings originated by board units. If nodes are not participating and communicating then it is difficult to achieve coordination and hence enhance network performance. Various researchers have proposed cluster based schemes $[1][2][3][4][5][6]$. Collision avoidance is necessary for smooth function of system [8][9][10][11][12] have presented collision avoidance based scheme to enhance network performance. Agent based approaches were highlighted in [13][14][15]. Trust establishment and security is crucial feature [16][17][18][19] threw light on these aspects and presented approaches for resolving these issues.[20][21][22]presented overview of routing protocols prevailing in VANETs
Cooperation among nodes is primary feature for attaining effective communication. Nodes may become non cooperative because of various reasons. If driver is not following instructions and alerts, reason may me that he or she is an amateur driver and should not be allowed to drive vehicle. Movements of steering wheel, brakes, speed and acceleration are the important factors that could determine the reasons of instruction violation by vehicles. If alerts and warnings are executed by vehicles they will move in required direction. The violation of rules is determined by a special in proposed work.

Synchronization is also achieved in VANET if desired information is timely shared by all the participating nodes. To achieve this task hierarchy based approach is deployed in proposed framework. Hierarchy table is constructed on the basis of which synchronization and communication priority is established.

In proposed framework it has been assumed that roadside units are fixed after every $\mathrm{n}$ kilometer approximately 1 to 2 kilometers. Objective of this approach is to achieve fast synchronization. For achieving this goal cluster file system is used to share copies of tables with all nodes. All the participating nodes are periodically updated with the status of neighboring nodes and hierarchy based approach is used to synchronize nodes and increase cooperation and network productivity. Inside the vehicles various alerts and warning messages are generated to assist driver to take safety actions so that accidents are avoided. These messages are categorized as follows.

- Warning Alerts: Drivers are assisted in driving safely by alerting them through warning signals. In present scenario with latest advancement in automobile designs on board units inside vehicles are designed and programmed to generate alert message. In proposed framework it has been assumed that sensors are embedded on brakes, steering wheel speedometers to assess their performance and check their movements. 
Agent software in sensors is programmed to record movements of vehicle and monitors the deviations found in movements. Warning signals are generated in the form of light indication on steering wheel, speedometer, wing mirrors and rear view mirrors and through graphic display on On Board Unit. These signals are of following types:

- Lane Change Warning (LCW): This message is generated to alert driver not to change lane. If direction of vehicle is toward right it intimates and generates alarm to instruct driver for not changing direction to left lane or vice versa. This movement is recorded by agent by determining direction of steering wheel.

- Collision Warning Alert (CWA): Forward collision could be avoided if agents generate warning alert for vehicle moving with high speed with chances of crash or collision.

- Intersection movement Warning (IMW): This warning is originated to alert vehicle to take right path. Intersection points on road are more prone to accidents. If driver is assisted to take right movement on intersections it could add to road safety.

- Turn Assistance Warning (TAW): This alert is generated to assist drivers to take appropriate turn. These warnings suggestions suggest drivers to take appropriate path as per information traffic information received from forward node.

- Safety Alerts (SA): Safety alerts are generated to enhance vehicles as well as its neighboring vehicle's safety. Safety warnings may include road conditions and weather forecast that are generated by sensors embedded in Road side Units. This information is communicated by roadside communicator agents.

- Nearby facility alerts (NFA): Nearby facility information is sometimes urgently required by vehicle and it becomes quite difficult to reach these facilities if their location information is not provided to moving vehicles. Road side units communicate this information to moving vehicles.

- Suggestion alerts (SA): Apart from safety and warning alerts, the proposed framework facilitates vehicles with suggestion alerts so that they can opt right path. This signal is generated through road map displayed on On Board units. Vehicle could move on that path where traffic is less and which is shorter. It shows various path options and benefits as to why that path should be chosen through display units.

\section{PROPOSED FRAMEWORK}

The proposed framework is divided into two phases. Agent based Vehicle to vehicle ( $\mathrm{V}$ to $\mathrm{V}$ ) communication phase and agent based Vehicle to Roadside Unit communication phase. Effective coordination and synchronization will be achieved by communication of these two phases.

\subsection{Phase I: Agent based Vehicle to Vehicle communication}

For making agent based vehicle to vehicle communication various agents have been inculcated in vehicles. These agents utilize their own information as well as information received from roadside unit. In proposed framework it has been assumed that following agents are embedded at various parts of automobile. This phase consists of two sub phases: Intra vehicle

\subsubsection{Intra vehicle communication: vehicle}

There are various sensors embedded within vehicle. These are equipped with programmed agents for interaction and recording movements of vehicle. In proposed work it has been assumed that every node will maintain some table with respect to its neighboring nodes. For instance there is a set of $\mathrm{n}$ nodes

$\mathrm{V}_{\mathrm{n}}=\left\{\mathrm{V} 1, \mathrm{~V} 2, \mathrm{~V}_{3}, \mathrm{~V}_{4} \ldots \ldots \ldots \mathrm{V}_{\mathrm{n}}\right\}$. Every $\mathrm{i}^{\text {th }}$ node will maintain all the tables with respect to its neighbor vehicles. Following tables are maintained in every vehicle's database.

1) NeighborExpected_table

2) NeighborActual_Table

3) Decision_Table

4) Vehicle_Grading_Table

1) NeighborExpected_table: This table is maintained by Vehicular performance agent (VPA) embedded in every node in VANET with respect to all neighbor nodes at specific hop distance. Information regarding neighbor nodes is stored and maintained by every node in this table. In this framework four situations have been described where expected speed and distance values of neighbor nodes are shown in NeighborExpected_table.

2) NeighborActual_Table :This table records actual speed of vehicles by Vehicular performance agent (VPA) and distance with neighbor nodes under various circumstances. Values stored in this table are used to calculate actual deviations. This table is stored in VPA of every neighbor node.

3) Decision_Table :Decision table is described by Vehicular performance Evaluation agent (VPEA) after analyzing the deviations after comparing actual values and expected values. It is important that vehicle should follow the decision for safe and smooth drive. After matching expected values with actual values obtained from vehicle, VPA evaluates the difference and provides decision for smooth driving of vehicle. For instance if it is found that there is danger alarm at lane change then vehicle has to control speed and stop its steering wheel rotation. Such decisions are taken by VPEA to control the movements of vehicle after analyzing its neighbors. It indicates to its own vehicle that neighbor is coming at particular speed at a specific distance and what is expected behavior of vehicle in that situation. 
4) Vehicle Grading Table

Vehicle Grading table is maintained by VPEA at every vehicle to grade vehicle performance by finding whether vehicle is following the decision given by Decision_table or not. If vehicle is following indications generated by VPEA then grade is $\alpha$ given to vehicle and if not followed $\beta$ is grade for that situation. $\mathrm{d}$ by agents inside the vehicle to assess the vehicle performance. Following steps are performed:

- Every ith node will broadcast hello message to all the nearest neighbor nodes.

- Neighbor nodes will reply back to ith node and will send their consent to share information.

- All the nearest neighbors will then share some tables and will maintain copies of all four tables in context of

Table 1. NeighborExpected_Table

\begin{tabular}{|c|c|c|c|c|c|c|c|}
\hline \multirow{3}{*}{$\begin{array}{l}\text { Neighbor } \\
\text { Nodes }\end{array}$} & \multirow{3}{*}{$\begin{array}{l}\text { Hop } \\
\text { Distance }\end{array}$} & \multirow{2}{*}{\multicolumn{3}{|c|}{ Expected_Distance }} & \multicolumn{3}{|c|}{ Expected_speed } \\
\hline & & & & & \multicolumn{3}{|c|}{ Density_level } \\
\hline & & Safe> & Alert & Danger & High Density & Average Density & Low Density \\
\hline Collision & 1 & 10 & $5-10$ & $3-5$ & Less than 40 & $40-60$ & $60-70$ \\
\hline Lane change & $1-3$ & 20 & $15-20$ & $10-15$ & Less than 40 & $40-60$ & $>=60$ \\
\hline Turn Assistance & 1 & 20 & $10-20$ & $5-10$ & Less than 40 & $40-50$ & $50-60$ \\
\hline Intersection & 1 & 20 & $10-20$ & $8-10$ & Less than 30 & $40-50$ & $50-70$ \\
\hline
\end{tabular}

Table 2 NeighborActual_Table

a particular neighbor.

\begin{tabular}{l|l|l|l|l|l|l|}
\cline { 2 - 7 } $\begin{array}{l}\text { These } \\
\text { tables } \\
\text { created } \\
\begin{array}{l}\text { and } \\
\text { maintaine }\end{array}\end{array}$ & V1 & Vehicle_Tags & Hop Distance & Actual_Distance & Actual_speed & Density \\
\cline { 2 - 8 } & V2 & V_LEFT1 & 1 & 8 & 60 & High \\
\cline { 2 - 8 } & V4 & V_Back1 & 1 & 1 & 60 & Low \\
\cline { 2 - 7 } & V_Forwad1 & 1 & 10 & 80 & Average \\
\hline
\end{tabular}

Table 4. Vehicle_Grading Table

\begin{tabular}{|c|c|c|c|c|c|c|c|}
\hline \multirow{2}{*}{$\begin{array}{l}\text { Neighbor } \\
\text { Nodes }\end{array}$} & \multirow[t]{2}{*}{ Vehicle_Tags } & \multicolumn{5}{|c|}{ Decision followed $V_{i}$} & \multirow[t]{2}{*}{ Grade } \\
\hline & & Signal & $\begin{array}{l}\text { Brake_move } \\
\text { _update }\end{array}$ & $\begin{array}{l}\text { Steering_Whl_Rota } \\
\text { tion_update }\end{array}$ & Speed_update & Distance_update & \\
\hline \multicolumn{8}{|c|}{ Turn Assistance } \\
\hline V1 & V_LEFT1 & DANGER & No_move & Rotate_Left & Speed_Reduce & Distance_Increased & $\beta$ \\
\hline $\mathrm{V} 2$ & V_Back1 & SAFE & No_move & Rotate_Left & Speed_same & Distance_Same & $\alpha$ \\
\hline V3 & V_Forwad1 & ALERT & Pushed & Rotate_Left & Speed_same & Distance_Same & $\beta$ \\
\hline $\mathrm{V} 4$ & V_Right1 & DANGER & Pushed & Rotate_Left & Speed_normal & Distance_Increased & $\alpha$ \\
\hline \multicolumn{8}{|c|}{ Collision } \\
\hline V1 & V_LEFT1 & DANGER & Pushed & No_rotate & Speed_red & Distance_Increased & $\alpha$ \\
\hline V2 & V_Back1 & SAFE & No_move & No_rotate & Speed_same & Distance_Same & $\alpha$ \\
\hline V3 & V_Forwad1 & ALERT & Pushed & No_rotate & Speed_Reduce & Distance_Increased & $\alpha$ \\
\hline V4 & V_Right1 & DANGER & Pushed & No_rotate & Speed_Reduce & Distance_Increased & $\alpha$ \\
\hline \multicolumn{8}{|c|}{ Lane change } \\
\hline V1 & V_LEFT1 & DANGER & Pushed & Rotate_Left & Speed_same & Distance_Same & $\beta$ \\
\hline $\mathrm{V} 2$ & V_Back1 & SAFE & No_move & Rotate_Left & Speed_same & Distance_Same & $\alpha$ \\
\hline V3 & V_Forwad1 & ALERT & Pushed & Rotate_Left & Speed_Reduce & Distance_Increased & $\alpha$ \\
\hline $\mathrm{V} 4$ & V_Right1 & DANGER & Pushed & Rotate_Left & Speed_Reduce & Distance_Increased & $\alpha$ \\
\hline \multicolumn{8}{|c|}{ Intersection } \\
\hline V1 & V_LEFT1 & DANGER & Pushed & Rotate_Left & Speed_Reduce & Distance_Increased & $\alpha$ \\
\hline V2 & V_Back1 & $\begin{array}{l}\text { SAFE } \\
\end{array}$ & No_move & Rotate_Left & Speed_same & Distance_Same & $\beta$ \\
\hline V3 & V_Forwad1 & ALERT & Pushed & Rotate_Left & Speed_same & Distance_Same & $\beta$ \\
\hline $\mathrm{V} 4$ & V_Right1 & DANGER & Pushed & Rotate_Left & Speed_Reduce & Distance_Increased & $\alpha$ \\
\hline
\end{tabular}


International Journal of Computer Applications Technology and Research

Volume 5- Issue 5, 281 - 291, 2016, ISSN:- 2319-8656

\begin{tabular}{|c|c|c|c|c|c|c|c|c|c|}
\hline \multirow{2}{*}{$\begin{array}{l}\text { Neighbor } \\
\text { Nodes }\end{array}$} & \multirow{2}{*}{$\begin{array}{l}\text { Vehicle } \\
\text { _Tags }\end{array}$} & \multirow{2}{*}{$\begin{array}{l}\text { Hop } \\
\text { Distance }\end{array}$} & \multirow{2}{*}{$\begin{array}{l}\text { Distance } \\
\text { alarm }\end{array}$} & \multirow{2}{*}{$\begin{array}{l}\text { Speed_ } \\
\text { level }\end{array}$} & \multirow[t]{2}{*}{ Density } & \multicolumn{4}{|c|}{ Table 3. Decision Table } \\
\hline & & & & & & Signal & Brake_move & Steering_Whl_Rotation & Speed \\
\hline \multicolumn{6}{|c|}{ Turn Assistance } & \multicolumn{4}{|c|}{ Left_Turn/Right_Turn } \\
\hline V1 & V_LEFT1 & 1 & Danger & High & High & DANGER & Pushed & No_Rotate & Reduce \\
\hline V2 & V_Back1 & 1 & Safe & Normal & Low & SAFE & No_move & Rotate_Left/Rotate_Right & No_change \\
\hline V3 & V_Forwad1 & 1 & safe & High & Average & ALERT & Pushed & Rotate_Left/Rotate_Right & Reduce \\
\hline V4 & V_Right & 1 & Danger & High & Low & DANGER & Pushed & Rotate_Left/Rotate_Right & Reduce \\
\hline \multicolumn{6}{|l|}{ Collision } & \multicolumn{4}{|c|}{ Left_Turn/Right_Turn/Foward } \\
\hline V1 & V_LEFT1 & 1 & Alert & High & High & DANGER & Pushed & No_Rotate & Reduce \\
\hline $\mathrm{V} 2$ & V_Back1 & 1 & Safe & Normal & Low & SAFE & No_move & Rotate_Left/ Right/No_Turn & No_change \\
\hline V3 & V_Forwad1 & 1 & Safe & High & Average & ALERT & Pushed & Rotate_Left/Right/No_Turn & Reduce \\
\hline V4 & V_Right1 & 1 & Alert & High & Low & DANGER & Pushed & Rotate_Left/ Right/No_Turn & Reduce \\
\hline \multicolumn{6}{|c|}{ Lane change } & \multicolumn{3}{|c|}{ Left_Lane/Right_Lane } & \\
\hline V1 & V_LEFT1 & 1 & Danger & High & High & DANGER & Pushed & No_Rotate & Reduce \\
\hline V2 & V_Back1 & 1 & Safe & Normal & Low & SAFE & No_move & Rotate_Left/Rotate_Right & No_change \\
\hline V3 & V_Forwad1 & 1 & Safe & High & Average & ALERT & Pushed & Rotate_Left/Rotate_Right & Reduce \\
\hline V4 & V_Right1 & 1 & Danger & High & Low & DANGER & Pushed & Rotate_Left/Rotate_Right & Reduce \\
\hline \multicolumn{8}{|c|}{ Intersection } & \multicolumn{2}{|l|}{$\begin{array}{l}\text { Left_Turn/ } \\
\text { Right_Turn/Forward }\end{array}$} \\
\hline V1 & V_LEFT1 & 1 & Danger & High & High & DANGER & Pushed & No_Rotate & Reduce \\
\hline $\mathrm{V} 2$ & V_Back1 & 1 & Safe & Normal & Low & SAFE & No_move & Rotate_Left/Right/No_Turn & No_change \\
\hline V3 & V_Forwad1 & 1 & Safe & High & Average & ALERT & Pushed & Rotate_Left/Right/No_Turn & Reduce \\
\hline V4 & $\overline{\text { V_Right1 }}$ & 1 & Danger & High & Low & DANGER & Pushed & Rotate_Left/Right/No_Turn & Reduce \\
\hline
\end{tabular}

Following steps are followed in detail for performing tasks of this framework:

1) Every node maintains NeighborExpected_Table with respect to its neighbor nodes. Given below is neighbor expected table of node $\mathrm{V}_{\mathrm{I}}$ for all neighbor nodes surrounding $\mathrm{V}_{\mathrm{i}}$

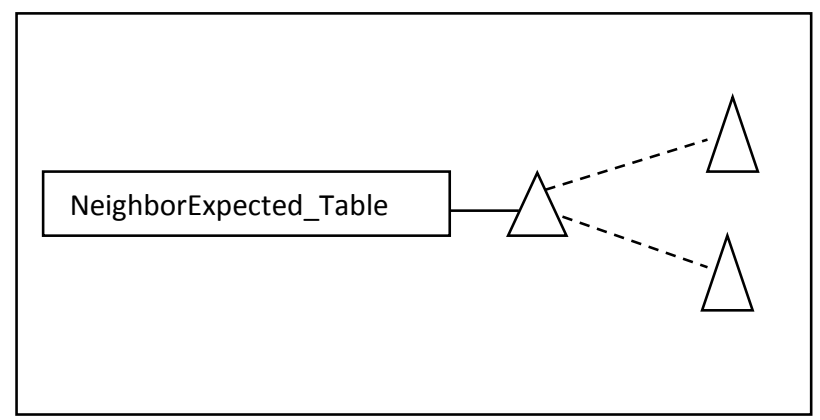

Figure1. NeghborExpected_Table at VCi

2) Node $V_{i}$ send empty copy of NeighbourActual_Table to all the $\mathrm{V}_{\mathrm{n}-\mathrm{i}}$ neighbor nodes to get actual value of neighbor performance. Neighbor nodes after filling values send NeighborActual_Table to $V_{i}$ node.

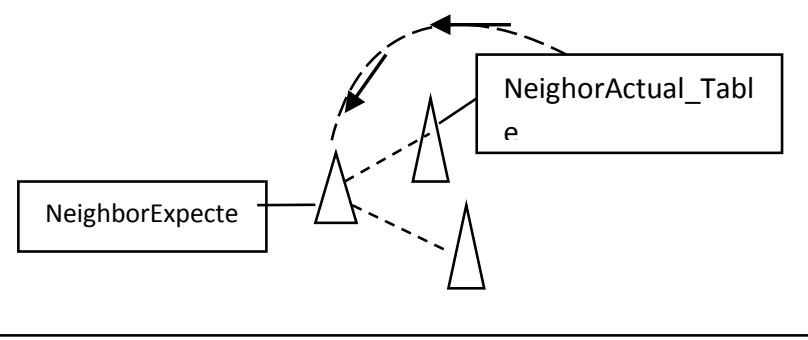

Figure 2. NeighbourActual_Table Sbmission

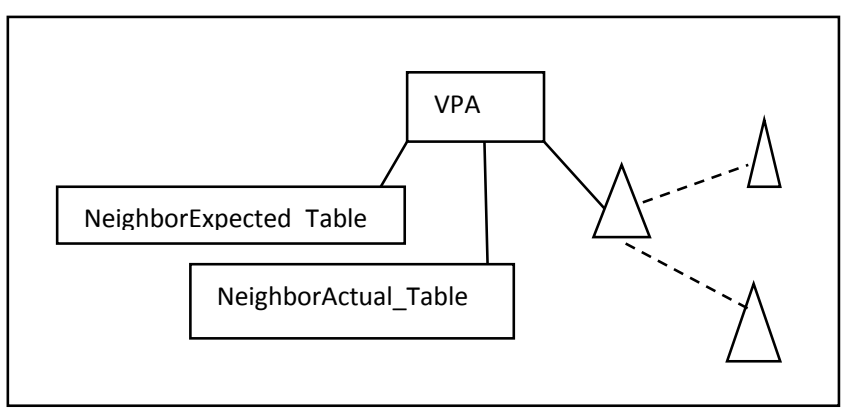

Figure. 3 NeighborActual \& Expected Table at VPA

The above figure shows two tables maintained at $\mathrm{V}_{\mathrm{i}}$ with respect to all $\mathrm{V}_{\mathrm{n} \text {-i.. }}$ All the nodes are equipped with a copy of 
NeighborExpected_Table and NeighborActual_Table received from rest of the neighbor nodes.

3) These two tables are registered at Vehicle Performance agent of vehicle and submitted to Vehicular performance evaluation agent to compare the values of two tables and decide what action vehicle has to follow.

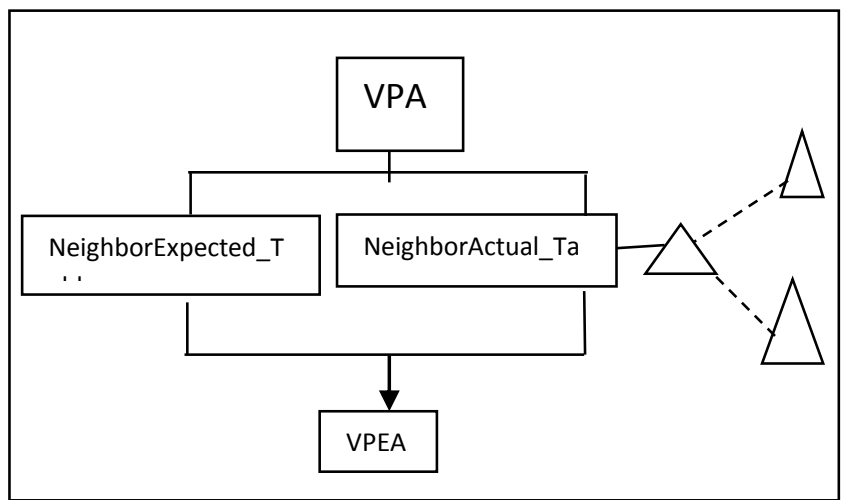

Figure 4. Table submission at VPEA

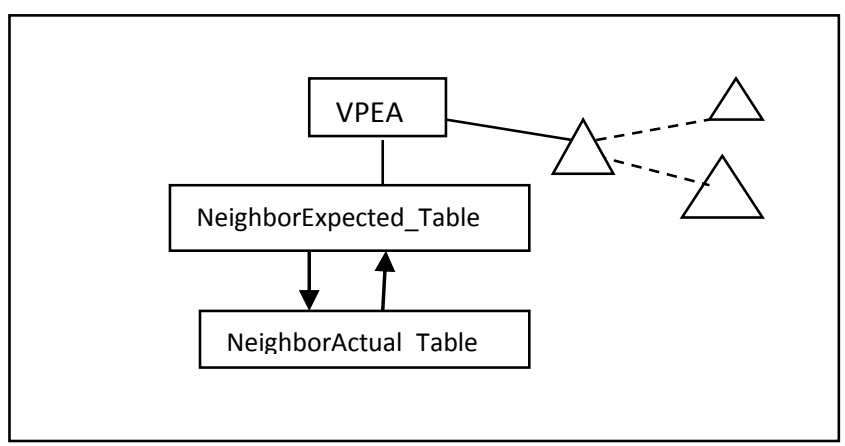

Figure 5. Comparison of Expected and Actual

4) After comparing the values of two tables vehicle performance evaluation agent finds grade of ith vehicle on the basis of fact that whether it has followed the decision or not. It assigns grade in Vehicle Grading table by assessing the performance of vehicle as per decision given by VPEA.

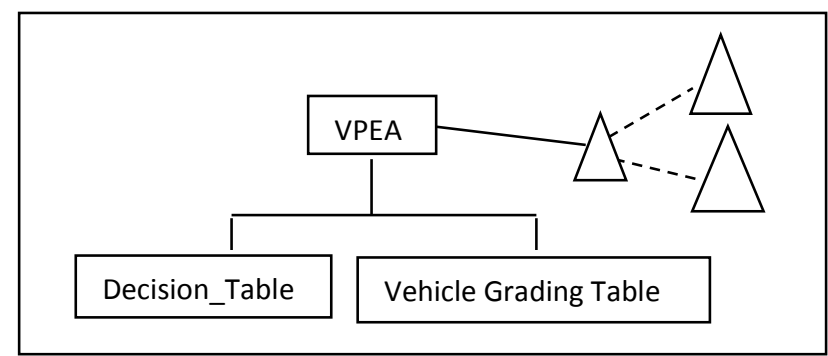

Figure 6. Decision and Vehicle Grading Table

The following algorithms are implemented Vehicle performance evaluation agent (VPEA): This agent is responsible to monitor and assess performance of vehicle by counting the number of times it has obeyed and followed the instructions and alerts. A vehicle is said to be honest and obeying rules if its brake state, steering wheel angle, speed limit changes as required by warning alerts. These alerts are originated in the form of electronic light signals on steering wheel, electronic brakes light and graphic display on On Board Units.

Main responsibilities of VPEA are:

1) To compare the actual movement of parts of vehicle with expected movements and find the deviations.

2) To grade a vehicle on basis of its performance.

Input: NeighborExpected_Table, NeighborActual_Table

Output: Decision_Table_VCi

$\{$ for $(i=n-i, n)$

\{

do

\{

get(density_level(expected_speed),Distance_parameters( expected_distance) $) \leftarrow$ NeighborExpected_Table

get(actual_speed,actual_distance,density $\leftarrow$ NeighborActual_Table

\}

If $\left(\left(\right.\right.$ Turn_assistance $\left.\left(\mathrm{VC}_{\mathrm{i}}\right)\right) \|\left(\right.$ Lane_change $\left.\left(\mathrm{VC}_{\mathrm{i}}\right)\right) \|(\mathrm{Collosion}(\mathrm{V}$ $\left.\left.\mathrm{C}_{\mathrm{i}}\right)\right) \|\left(\right.$ Intersection $\left.\left.\left(\mathrm{VC}_{\mathrm{i}}\right)\right)\right)$

\{

If $(($ actual_speed $>$ expected_speed(Density_level) $) \& \&($ atual_distance $<\exp$ ected_distance(Distance_parameters=Danger))

\{

Speed_level=High

Distance_alarm=Danger

\{

Set Signal= DANGER

set speed=speed_reduce

Set distance $=$ distance_increase

Set brake_move=pushed

\}

\}

If((actual_speed>expected_speed(Density_level)\| (atual_distance<expected_distance(Distance_parameters=Aler t))

\{

Speed_level=High

Distance_alarm=Alert

\{ 
International Journal of Computer Applications Technology and Research

Volume 5- Issue 5, 281 - 291, 2016, ISSN:- 2319-8656

Set Signal= ALERT

set speed=speed_reduce

Set distance $=$ distance_increase

Set brake_move $=$ pushed

\}

If $((($ actual_speed $>$ expected_speed(Density_leve $) 1) \|$

(atual_distance >=expected_distance(Distance_parameters)))

\{

Speed_level=high

Distance_alarm=safe

\{

Set Signal $=$ alert

set speed=speed_reduce

Set distance=distance_same

Set brake_move=pushed

\}

If $((($ actual_speed <expected_speed(Density_level) $) \|$

(atual_distance $<=$ expected_distance(Distance_parameters))

\{

Speed level=normal

Distance_alarm=alert/danger

\{

Set Signal= alert/danger

set speed=speed_reduce

Set distance $=$ distance_same

Set brake_move=pushed

\}

\}

If $(($ actual_speed $<=$ expected_speed $) \& \&$

(atual_distance $>=$ expected_distance(Distance_parameters $=$ safe $)$ )

\{

Speed_level=Normal

Distance_alarm $=$ safe

\{

Set Signal $=$ safe

set speed=speed_same

Set distance $=$ distance_same

Set brake_move=no_move

\}

\}

If left_turn( $\left.\mathrm{VC}_{\mathrm{n}-\mathrm{i}}\right)$

Set steering_whl_rotation $\left(\mathrm{VC}_{\mathrm{i}}\right)=$ rotate_left

If right_turn $\left(\mathrm{VC}_{\mathrm{n}-\mathrm{i}}\right)$

Set steering_whl_rotation $\left(\mathrm{VC}_{\mathrm{i}}\right)=$ rotate_right

If no_turn $\left(\mathrm{VC}_{\mathrm{n}-\mathrm{i}}\right)$

Set steering_whl_rotation $\left(\mathrm{VC}_{\mathrm{i}}\right)=\mathrm{No} \_$rotate

Create decision_table_ $\mathrm{VC}_{\mathrm{i}}$

put

(speed_level $\left(\mathrm{VC}_{\mathrm{i}}\right)$,Distance_alarm $\left(\mathrm{VC}_{\mathrm{i}}\right)$,signal $\left(\mathrm{VC}_{\mathrm{i}}\right)$, brake_m $\operatorname{ove}\left(\mathrm{VC}_{\mathrm{i}}\right)$,steering_whl_rotation $\left.\left(\mathrm{VC}_{\mathrm{i}}\right)\right) \rightarrow$ Vehicle_Grading_Ta ble

update Decision_table_VCI
Vehicle performance in terms of vehicle grade could be evaluated as follows:

Input: Decision_Table_VC

Output: Vehicle_Grading_Table

for $(i=n-i, n)$

\{

If(signal=danger)

\{

If

((brake_move!=brake_move_update)||

(Steering_Whl_Rotation!=Steering_Whl_Rotation_update)||

(actual_speed!=speed_update)

||(actual_distance!=distance_update))

Grade $=\beta$

ElseIf ((brake_move!=brake_move_update) \|

(Steering_Whl_Rotation!=Steering_Whl_Rotation_update)or (actual_speed!=speed_update)

(actual_distance!=distance_update))

Garde $=\alpha$

OR

If(signal=alert)

\{

If ((brake_move!=brake_move_update)

(Steering_Whl_Rotation!=Steering_Whl_Rotation_update)or (actual_speed!=speed_update)

(actual_distance!=distance_update))

Grade $=\beta$

ElseIf((brake_move!=brake_move_update)

(Steering_Whl_Rotation!=Steering_Whl_Rotation_update)\| (actual_speed!=speed_update)

||(actual_distance!=distance_update) $)$

Grade $=\alpha$

\}

If $($ Signal $=$ Safe $)$

\{

If((brake_move!=brake_move_update) $\|$

(Steering_Whl_Rotation!=Steering_Whl_Rotation_update) $\|($ a ctual_speed!=speed_update)

(actual_distance!=distance_update))

Grade $=\beta$ 
Elseif((brake_move!=brake_move_update)\|

(Steering_Whl_Rotation!=Steering_Whl_Rotation_update) $\|($ a ctual_speed!=speed_update)

(actual_distance!=distance_update))

Grade $=\alpha\}$

Update Vehicle_Grading_Table

\}

Vehicle communicator agent: This agent communicates status of vehicle performance with neighbor nodes. Nodes near to a particular vehicle follow actions to enhance road safety.

\subsubsection{Master vehicle communicator}

Master vehicle communicator is cluster head. For first iteration MVC is elected randomly. But for iterations MVC is calculated on the basis of some attributes. MVC election criteria are set by Roadside Unit on the basis of parameters received from MVC. Vehicle communicator of every vehicle calculate grade of vehicle and that information is communicated to other vehicles and MVC records that information and when it leaves the cluster it submit all the current updated information to roadside unit after every $n$ kilometers. The table created by MVC has following attributes:

1) Vehicle grade: Vehicle grade is retrieved from Vehicle_Grading_table and this grade is considered as one of the contributing factor for determining the rank of vehicle. The entire vehicles have to submit this report to MVC. In case vehicle is not submitting the report that vehicle is declared as non participating node and its rank in the hierarchy table get diminished.

2) Participation history: participation history is determined by counting the number of times vehicle has participated in communication and it has followed the instructions. It is counted on the basis of vehicle_grade that is communicated by Vehicle communicator agent. Participation_history_table is maintained at MVC and it record various attributes that are considered in Participation_history_table.

Participation_History_Table Table 5. is created to determine participation value of vehicle. Participation value is calculated on the basis of Grades achieved by vehicles in different situation. This table is prepared on the basis of vehicle grade from vehicle_grade_table. Alpha is considered as positive contribution and Beta as negative. Participation_History_Table calculates total number of positive and negative contributions of all participating vehicles and enters highest number of contribution in the participation value column.

3) Path selection: Path is selected by a vehicle on the basis of suggestions that it has received from forward vehicle.
The vehicle ahead gives path selection suggestion to vehicles following that vehicle. For a particular cluster MVC provides details of safe paths and smooth paths to road side units and RSU agents communicate that information to new MVC that is elected for next cluster and this process goes on.

In figure 7 vehicle nodes are connected through communication channel. MVCA of one cluster $\mathrm{C} 1$ is communicating with MVCA of another cluster $\mathrm{C} 2$ and sending safe and smooth path information to previous cluster. MVCA of C1 submits information regarding path to nearest RSU while leaving the cluster. RSU uses this message to determine whether nodes are following path or not. Safe and smooth path followed by a particular vehicle is used as one of the parameter for assessing performance of vehicle by RSU.

Table 6 shows grading of path followed by vehicles and this Path_Follow_Grade is helpful in determining whether a vehicle is following a path or not. In case a vehicle is not following path its value will be degraded in final ranking of vehicles.

4) Energy level: Energy level of nodes will also be considered as one of the parameter to calculate hierarchy rank of a particular node.

MVC of each cluster generates a table that contains overall performance of vehicles in cluster in terms of vehicle grade, participation history, path selection and energy level. This table is submitted to nearest RSU and MVC of preceding clusters and this information is circulated by MVC to all the vehicles of cluster.Table 7 is MVC table given below.

\subsection{Phase II: Agent Based Vehicle to roadside communication}

Roadside Unit: It has been assumed in this framework that roadside unit is installed after every n kilometers and Road side unit has agent software embedded inside it that is programmed to store information that is submitted by Master Vehicle Communicator agent. MVCA of every cluster submits some information to nearest RSU at the time of leaving a cluster. Roadside Unit agent maintains a table in which it records information regarding all the vehicles in cluster. MVC submits details of MVC_table to the road side unit after every 1 or 2 kilometers. 
Table 4.5. Participation_History Table

\begin{tabular}{|c|c|c|c|c|c|c|c|}
\hline \multirow{2}{*}{$\begin{array}{c}\text { Neighbor } \\
\text { Nodes }\end{array}$} & Vehicle_Tags & \multicolumn{5}{|c|}{ Vehicle Grade } & Turn \\
\cline { 3 - 7 } & & Signal & $\begin{array}{c}\text { Collision } \\
\text { Assistance }\end{array}$ & Lane change & $\begin{array}{c}\text { Intersection } \\
\text { Participation } \\
\text { Value }\end{array}$ \\
\hline V1 & V_LEFT1 & DANGER & $\beta$ & $\alpha$ & $\beta$ & $\alpha$ & 2 \\
\hline V2 & V_Back1 & SAFE & $\alpha$ & $\alpha$ & $\alpha$ & $\beta$ & 3 \\
\hline V3 & V_Forwad1 & ALERT & $\beta$ & $\alpha$ & $\alpha$ & $\alpha$ & 4 \\
\hline V4 & V_Right1 & DANGER & $\alpha$ & $\alpha$ & $\alpha$ & $\alpha$ & 2 \\
\hline
\end{tabular}

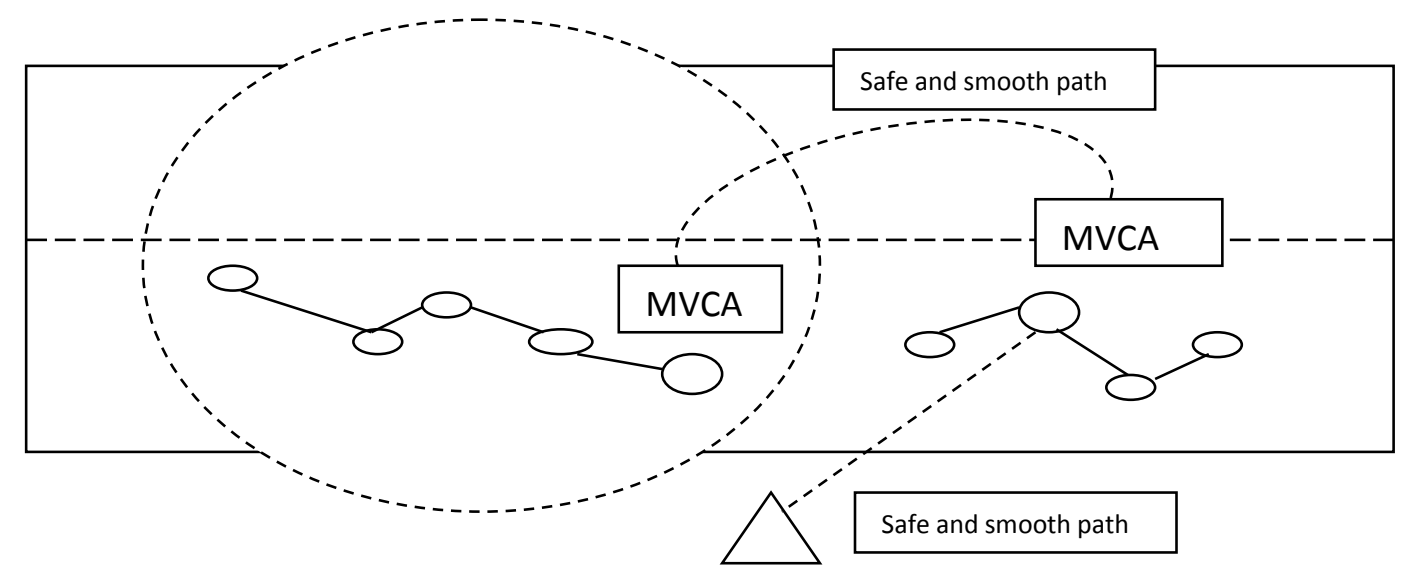

Figure 4.7.Path Selection by MVC

Table 6. Path_Follow Table

\begin{tabular}{|c|c|c|c|c|c|}
\hline $\begin{array}{c}\text { Neighbo } \\
\text { r Nodes }\end{array}$ & Vehicle_Tags & Smooth_path & Safe_Path & Actual_Path & Path_Follow_Grade \\
\hline V1 & V_LEFT1 & $\begin{array}{l}\text { V2_C1 } \\
\text { V3_C1 } \\
\text { V5_C1 } \\
\text { V2_C2 } \\
\text { V4_C2 }\end{array}$ & $\begin{array}{l}\text { V2_C1 } \\
\text { V3_C1 } \\
\text { V5_C1 } \\
\text { V2_C2 } \\
\text { V4_C2 }\end{array}$ & $\begin{array}{l}\text { V2_C1 } \\
\text { V3_C1 } \\
\text { V5_C1 } \\
\text { V2_C2 } \\
\text { V4_C2 }\end{array}$ & $\alpha$ \\
\hline $\mathrm{V} 2$ & V_Back1 & $\begin{array}{l}\text { V1_C2 } \\
\text { V3_C2 } \\
\text { V4_C3 } \\
\text { V5_C3 }\end{array}$ & $\begin{array}{l}\text { V1_C2 } \\
\text { V2_C2 } \\
\text { V4_C3 } \\
\text { V5_C3 }\end{array}$ & $\begin{array}{l}\text { V1_C2 } \\
\text { V3_C2 } \\
\text { V5_C3 } \\
\text { V5_C4 }\end{array}$ & $\beta$ \\
\hline
\end{tabular}

Table 7. MVC Table

\begin{tabular}{|c|c|c|c|c|c|}
\hline $\begin{array}{c}\text { Cluster } \\
\text { Nodes }\end{array}$ & Vehicle_Tags & Vehilce_grade & Participation_History & Path_follow & Energy_level \\
\hline V1 & V_LEFT1 & $\beta$ & 2 & $\alpha$ & 65 \\
\hline V2 & V_Back1 & $\alpha$ & 3 & $\beta$ & 65 \\
\hline V3 & V_Forwad1 & $\beta$ & 2 & $\beta$ & 40 \\
\hline V4 & V_Right1 & $\alpha$ & 4 & $\alpha$ & 60 \\
\hline
\end{tabular}


Hierarchy Determination and MVC Election: On the basis of grade, participation history, path selection and energy level of vehicle hierarchy is determined. There are many benefits of establishing hierarchy ranking of vehicles of VANET. The vehicle having highest rank will have capability to become Master Vehicle communicator or cluster head only. Because that vehicle is efficient in all aspects and will be able to replicate and share copies of updated information more efficiently. This will be beneficial in achieving data synchronization and process synchronization also as all the nodes will be associated very quickly and easily with fast availability of updated information.

Table 8 . Hierarchy_Table

\begin{tabular}{|c|c|c|c|c|c|}
\hline $\begin{array}{c}\text { Clus } \\
\text { ter } \\
\text { Nod } \\
\text { es }\end{array}$ & $\begin{array}{c}\text { Vehilce_ } \\
\text { grade }\end{array}$ & $\begin{array}{c}\text { Participation } \\
\text { _History }\end{array}$ & $\begin{array}{c}\text { Path_sel } \\
\text { ection }\end{array}$ & $\begin{array}{c}\text { Energy_ } \\
\text { level }\end{array}$ & $\begin{array}{c}\text { Hierar } \\
\text { chy }\end{array}$ \\
\hline V1 & $\beta$ & 2 & $\alpha$ & 65 & 3 \\
\hline V2 & $\alpha$ & 3 & $\beta$ & 65 & 2 \\
\hline V3 & $\beta$ & 2 & $\beta$ & 40 & 4 \\
\hline V4 & $\alpha$ & 4 & $\alpha$ & 60 & 1 \\
\hline
\end{tabular}

This Hierarchy_table determines the priority of vehicles participating in the cluster. Hierarchy is determined on the basis of vehicle grade, its participation history, path selection and energy level.

This table is maintained at RSU on the basis of MVC. For example in above table vehicle V3 is the vehicle elected as master vehicle communicator for next cluster.

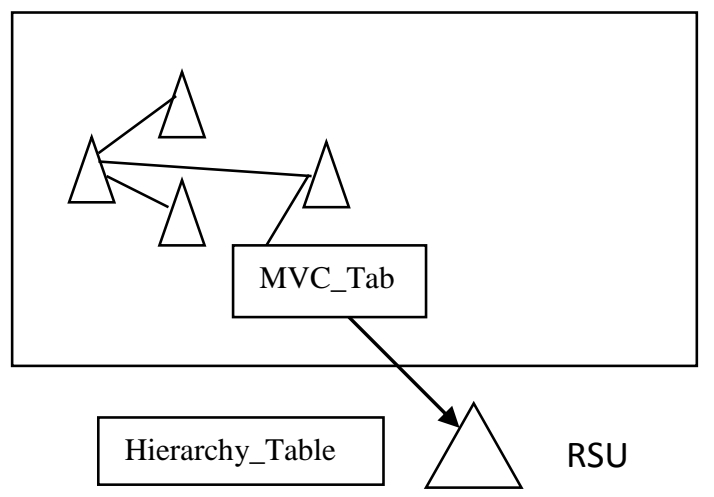

Figure 9. MVC_Table submission and Hierarchy_Table formation at RSU

Master Vehicle communicator submits MVC_Table to nearest RSU. It is not necessary that cluster reformation takes place or not. MVC_Table is submitted to every nearest RSU by Master Vehicle communicator. It is responsibility of RSU to elect the next Master Vehicle communicator on the basis of grades earned by vehicles participating in VANET. RSU determines hierarchy of participating vehicles on the basis of vehicle grade, participation history, path selection and energy level of nodes. Hierarchy table is updated whenever MVC_Table is submitted by MVC at RSU

\subsection{Phase III: Roadside Unit to Roadside unit communication}

RSU maintains copy of all the tables. Vehicle communicators Agent (VCA) of all vehicles submit details of all the four tables to MVC of that cluster. MVC submits all the tables to Roadside Units. Road side Units communicates this information to another RSU so that information about nearby facilities and warnings are communicated to nodes in cluster. All the RSUs receive information from previous RSU and update all the participating nodes with road conditions, Path conditions, nearby facility information, smooth and safe path updates.

\subsubsection{Motivation based approach:}

Motivation based approach is used in proposed framework to promote vehicles with low grade in hierarchy to participate actively and follow warnings and alerts suggested by VPEA. This process is followed as per below mentioned steps.

1) Roadside units asses performance of vehicles and generates hierarchy. This information is submitted to next nearest RSU e.g. V4 in Hierarchy_Table

2) If next RSU again maintain Hierarchy table and Value of V4 grade increases then V4 will get advantage in terms of discount on any nearby facility.

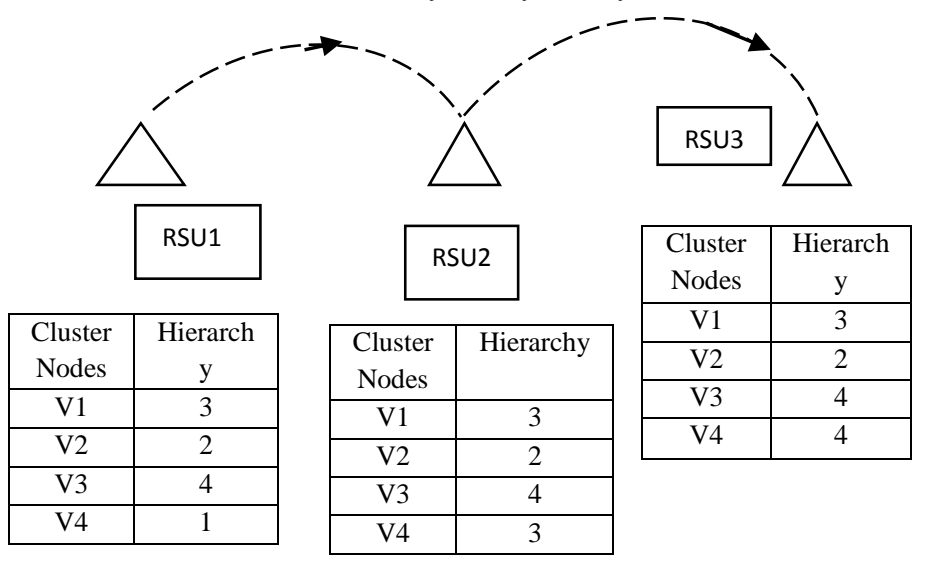

3) Hierarchy table is transmitted from one RSU to another and when it reaches last RSU3 value of V4 increases.

4) Increasing value of V4 indicates that V4 has started participating in VANET and additional benefits are given to V4. These benefits could be a discount at nearby facility. RSU3 will communicate to nearest restaurant, hospital, filling station or any other nearby facility to provide $10 \%$ discount to that vehicle.

5) If a vehicle is not responding and participating in communication and not following any alert or warning, 
then this report should be submitted to vehicle recovery agent (VRA) that resides with RSU.

6) VRA plays significant role in detecting problems with non-participating nodes. VRA analyzes Fuel level, vehicle grade that further consist of all aspects that have been considered in all the four tables, Hierarchy value and all the parameters that were actually utilized for its calculation. After analyzing all the factors it decides whether a node is selfish or needy.

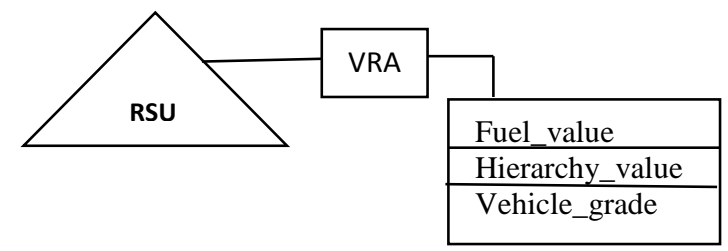

Figure 10. Vehicle_Recovery Agent

7) If a vehicle is rich in all aspects and not participating then the Vehicle_Status is selfish and if it is deficient in any of the three aspect then Vehicle_Status is needy and corrective measures are taken to improve the condition and status of that vehicle. Vehicle status is determined on the basis of Hierarchy_value and Fuel_Value

$$
\begin{aligned}
& \text { If } \quad\{(\text { Hiearchy_value=Low }) \\
& (\text { Fuel_Value }<=25 \%)\} \\
& \text { Then Vehicle_Status=Needy } \\
& \text { Else Vehicel_Status=Selfish. }
\end{aligned}
$$

This Vehicle_Status is reported by VRA to nearby road safety authorities and corrective measures are taken after checking driver details. VRA plays an important role in determining status of vehicle.

\section{CONCLUSION}

In this work various minor aspects have been presented that could play a significant role in determining the performance of vehicles. Road safety could only be enhanced if vehicles obey rules and run smoothly. In this multii agent based proposed framework vehicle performance is calculated on the basis of various movements. Agent embedded inside vehicles and roadside units are the main entities that enable fast communication among vehicles. Communication among vehicles are only effective if an individual vehicle is able to present its exact and accurate status to surrounding nodes.Agents inside vehicle are calculating vehicle's performance and communicating regularly with other vehicles and road side units. For communication to be effective it is necessary to improve both inter as well as intra vehicle communication. Our future task would be to implement this framework in JADE. After agent communication network performance will be verified in network simulator.

\section{REFERENCES}

[1] Xiaonan W. and Huanyan Q., 2013. "Constructing a VANET based on cluster chains," International Journal of Communication Systems,; doi:10.1002/dac.2484. 16.

[2] Venkataraman H., Delcelier R., and M.Muntean G., 2013. "A moving cluster architecture and an intelligent resource reuse protocol for vehicular networks," Wireless Net-works, Vol. 19, pp. 1881-1900.

[3] Tang X., Sun H., Sun L., Tan C., and Xu G, 2013. "Game theoretical approach for ad dissemination in cluster based VANETs," in Proceedings of IEEE International Conference on Signal Processing, Communication and Computing, pp. 1-6. 18.

[4] Schoch E., Kargl F., Weber M., and .Leinmuller T., 2008, "Communication patterns in VANETs," IEEE Communications Magazine, Vol. 46, pp. 119-125. 19.

[5] Hassanabadi B., Shea C., Zhang L., and Valaee S., 2014. "Clustering in vehicular ad hoc networks using affinity propagation," Ad-Hoc Networks, Vol. 13, , pp. 535-548. 20.

[6] Rawshdeh Y. Z. and Mahmud S. M., 2009. "Toward strongly connected clustering structure in vehicular adhoc networks," in Proceedings of the 70th IEEE Vehicular Technology Conference, pp. 1-5. 21.

[7] Girinath D. R. and Selvan S., 2013. "A novel hierarchical model for vehicular traffic regulation," Telecommunication Systems, Vol. 52, pp. 2101-2114

[8] Amudhavel J, et al. 2015.An robust recursive ant colony optimization strategy in VANET for accident avoidance (RACO-VANET). International Conference on Circuit, Power and Computing Technologies (ICCPCT); Nagercoil.. p. 1-6.

[9] Valdes-Vela M, Toledo-Moreo R, Terroso-Saenz F, Zamora-Izquierdo MA2013. An Application of a fuzzy classifier extracted from data for collision avoidance support in road vehicles. Journal of Engineering Applications of Artificial Intelligence.26(1):173-83.

[10] Milanes V, Perez J, Godoy J, Onieva E. 2012.A fuzzy aid rear-end collision warning/avoidance system. Journal of Expert Systems with Applications.; 39(10):9097-107.

[11] Amudhavel J, et al. . 2015.A krill herd optimization based fault tolerance strategy in MANETs for dynamic mobility. International Conference on Circuit, Power and Computing Technologies (ICCPCT); Nagercoil. p. 1-7.

[12] Wang L, Schmidt B, Nee AYC. 2013. Vision-guided active collision avoidance for human-robot collaborations. Journal of Manufacturing Letters.; 1(1):5-8

[13] Nadeem, S. Dashtinezhad, C. Liao, and L. Iftode, 2004."Traffic view: Trafficdata dissemination using carto-car communication,"ACM SIGMOBILE Mobile Computing and Communications Review, vol. 8, no. 3, pp. 6-19,

[14] Dietzel S., Bako B., Schoch E., and Kargl F., 2009. “A fuzzy logic based approach for structure-free aggregation in vehicular ad-hoc networks,"in Proc. ACM international workshop on Vehicular Inter-networking, New York, NY. 
[15] C. Sommer, O. K. Tonguz, and F. Dressler. "Traffic information systems: efficient message dissemination via adaptive beaconing," Communications Magazine, vol. 49 , no. 5, pp. 173-179, 201

[16] Raya M.and Hubaux J.P.,2005. "The Security of Vehicular Ad-hoc Networks," in 3rd ACM workshop on Security of Ad-hoc and sensor networks (SASN).

[17] Sapna S. Kaushik ,2013."Review Of Different Approaches For Privacy Scheme In Vanets" International Journal Of Advances In Engineering \& Technology.Vol. 5, Issue 2, pp. 356-363.

[18] John Moses S., Anitha Christy Angelin P. 2013. "Enhancing the Privacy through Pseudonymous Authentication and Conditional Communication in Vanets" Research Inventy: International Journal Of Engineering And Science Issn: 2278-4721, Vol. 2, Issue 7, Pp 45-49.www.Researchinventy.Com

[19] Jose Maria de Fuentes, Ana Isabel Gonzalez-Tablas, and Arturo Ribagorda, 2009. "Overview of Security issues in
Vehicular Ad Hoc Networks", Handbook of Research on Mobility and Computing,

[20] Chadha D., Reena, Vehicular Adhoc Network (VANETs): A Review, International Journal of Innovative Research in Computer and Communication Engineering, Vol. 3, Issue 3, March 2015.

[21] Zhang, Mingliu, and R. S. Wolff, 'Routing protocols for vehicular ad hoc networks in rural areas', Communications Magazine, IEEE, 46.11, pp.19-131, 2008.

[22] Khan, Imran, and Qayyum A., 2009.'Performance evaluation of AODV and OLSR in highly fading vehicular ad hoc network environments',INMIC IEEE 13th International Conference.

[23] Hashimi, Haider N, Kamalrulnizam Abu B, and Kayhan Zrar Ghafoor,2011. 'Inter-domain proxy mobile ipv6 based vehicular network',Network Protocols and Algorithms, vol.2, issue 4, pp. 1-15. 